\title{
Stress protocol and accuracy of myocardial perfusion imaging: Is it better to start from the end?
}

\author{
Marco Spadafora, MD, ${ }^{a}$ Marco Salvatore, $M D,{ }^{b}$ and Alberto Cuocolo, $M^{c}$ \\ ${ }^{a}$ Nuclear Medicine Unit, San Giuseppe Moscati Hospital, Avellino, Italy \\ ${ }^{b}$ IRCCS SDN, Naples, Italy \\ c Department of Advanced Biomedical Sciences, University Federico II, Naples, Italy
}

Received Mar 10, 2015; accepted Mar 10, 2015

doi:10.1007/s12350-015-0119-2

See related article, pp. 1114-1122

The strength of single-photon emission computed tomography (SPECT) myocardial perfusion imaging (MPI) is mainly due to the prognostic value also related with the choice of optimal therapeutic strategy. ${ }^{1-6}$ In the current era of comparative effectiveness and outcomes research, we are seeing a progressive shift from a diagnostic approach to a risk- and care costs-management of coronary artery disease (CAD). ${ }^{7}$ Recent technological advances in SPECT hardware and software, new stressors, and clinical protocols have improved the clinical impact and cost effectiveness of MPI. Particularly, the novel cadmium zinc telluride (CZT) SPECT cameras provide fast high-quality MPI and seem to allow for a more accurate evaluation of myocardial ischemia with lower administered doses. ${ }^{8}$ In this issue of the Journal, Gimelli et $\mathrm{al}^{9}$ assessed with a CZT camera the diagnostic accuracy of MPI for both pharmacologic and exercise stress test, also in patients undergoing sub-maximal exercise. They found an elevated accuracy of MPI, independently on both stress protocol and level of exercise stress testing. The topic is important, and the possibility that enhanced accuracy by CZT cameras could help to partially overcome the limitations of exercise level threshold, usually expressed as $85 \%$ of age-predicted maximal heart rate (HR), is of clinical relevance, expanding the indication of exercise test over pharmacologic stressor. However, there are some issues that should be taken into account.

Reprint requests: Alberto Cuocolo, MD, Department of Advanced Biomedical Sciences, University Federico II, Naples, Italy; cuocolo@unina.it

J Nucl Cardiol 2016;23:1123-7.

$1071-3581 / \$ 34.00$

Copyright (c) 2015 American Society of Nuclear Cardiology.

\section{THE CONCEPT “START FROM THE END”}

When there are in a pathway several possible options, it is often convenient to "start from the end," i.e., establish the goal of a procedure and then back links to choose the right options. Probably, the primary objective of MPI is risk stratification and consequently to guide clinical decision-making. For the assessment of myocardial ischemia, MPI must be performed in conjunction with a provocative test. Therefore, we are interested to gain global information of the tests, such as exercise electrocardiography (ECG), in which stressor is a not negligible part.

\section{CZT CAMERAS AND ACCURACY OF EXERCISE MPI}

Gimelli et $\mathrm{al}^{9}$ selected 475 subjects with anginal chest pain, with the mean age of 69 years. In this population, pre-test probability could be judged as high or intermediate if typical/definite or atypical/probable angina pectoris was present. ${ }^{10}$ Among 303 patients undergoing exercise, a high proportion $(69 \%)$ had submaximal test, the majority of them with $<75 \%$ of agepredicted maximal HR. In this large subgroup of patients, $62 \%$ terminated the exercise because of severe chest pain and/or significant ST segment depression. This clinical scenario, with high ischemic burden and clinical evidence of frequently low threshold-induced myocardial ischemia (angina and/or ST-segment depression), may account for the good sensitivity of MPI also at low level of HR. Therefore, it is conceivable that these findings are independent on the use of a novel SPECT camera. Yet, it is important to highlight that the data presented by Gimelli et $\mathrm{al}^{9}$ show an overall better detection of ischemia by exercise test, although often sub-maximal, compared with pharmacologic stress. In 
this regard, it should be also considered that intravenous dipyridamole at the conventional dose of $0.56 \mathrm{mg} \cdot \mathrm{kg}^{-1}$ body weight may not produce maximal coronary vasodilatation in all patients. ${ }^{11}$

It is likely that higher CZT resolution and sensitivity should be reflected in a balanced way on both exercise and pharmacologic stressors. Noteworthy, the authors demonstrate that in moderately sub-maximal exercise stress test, the novel camera maintains an excellent diagnostic power expanding the indication of exercise test. This conclusion could be linked to the group of patients with high ischemic burden. The key point "maintains an excellent diagnostic power in moderately sub-maximal exercise stress test' also deserves some consideration. In their study, the authors assessed CAD on the basis of a dichotomy parameter of ischemia (i.e., summed difference score $>$ or $<3$ ). In the setting: "start from the end" (the outcome), the extent and severity of stress-induced myocardial ischemia are not only diagnostic features, but also strong prognostic determinants. ${ }^{2,3}$ Can we be sure that an MPI with blunted HR response reveals the real degree of myocardial perfusion abnormalities? A possible underestimation of ischemia might lead to suboptimal diagnosis and risk stratification as well as to inappropriate patient management. $^{5,6}$ Therefore, if for diagnostic purposes, a test moderately sub-maximal could also be considered able to maintain high MPI sensitivity, then the impact on a more accurate evaluation based on quantitative data remains to be defined.

\section{MAXIMAL EXERCISE ECG AND MPI}

Despite the well-known limitations of the standard exercise ECG for diagnosing obstructive CAD, this approach continues to have an enormous prognostic value primarily based on measures of functional capacity, chronotropic response, exercise-induced angina, HR recovery, and ventricular ectopy. ${ }^{12}$ Exercise capacity alone is a powerful predictor of cardiovascular events $^{13}$ to the extent that some authors suggest that additional risk stratification with MPI might be eliminated in individuals who achieve $85 \%$ of their maximum predicted HR and 10 metabolic equivalents (METS) without ischemic ST-segment depression. ${ }^{14}$ Duvall et $\mathrm{al}^{15}$ proposed a protocol in which a patient would not receive tracer injection if adequate exercise is achieved without symptoms and ECG abnormalities, saving a substantial amount of time, radiation exposure, and cost.

Conflicting data are still present about the need for a maximal exercise test to maintain the diagnostic accuracy of MPI. ${ }^{16}$ In the milestone study of Iskandrian et $\mathrm{al},{ }^{17}$ the level of exercise affected the results of MPI in the detection of ischemia. However, in patients with two- or three-vessel CAD, the difference of vascular territories with perfusion defects was not statistically significant between patients with adequate and submaximal exercise. These findings seem to be in agreement with those reported by Gimelli et $\mathrm{al}^{9}$ on accuracy of sub-maximal MPI in groups with greater ischemic burden. In the study of Iskandrian et al, ${ }^{17} \mathrm{CAD}$ was considered to be present when there was $\geq 50 \%$ stenosis, whereas Gimelli et al $^{9}$ considered significant a threshold $\geq 70 \%$. This aspect could explain the differences in patients with less severe disease. Interestingly, in the study of Iskandrian et al, ${ }^{17}$ no patients with $<85 \%$ of predicted HR had a positive exercise test, and those with a positive exercise ECG were included in the group with adequate exercise end points. This confirms the importance of the selection methods. Nevertheless, both studies $^{9,17}$ mainly assessed the diagnostic sensitivity of MPI in CAD rather than the extent and severity of the stress-induced ischemia. It has also been shown that in patients with normal MPI, cardiac event rate is not related to any measured index of exercise stress, including peak HR, rate-pressure product, percent maximal-predicted HR, and peak METS, or Bruce stage achieved. ${ }^{18}$ Cardiac risk is linked with ischemia and independent of exercise HR level achieved, but this independence may not apply to patients with abnormal MPI and, more importantly, the need for revascularization might be underestimated if a poor HR response is achieved. ${ }^{18}$ These observations reflect, at least in part, an underestimation of underlying CAD by MPI. In the same patients, adenosine-induced perfusion abnormalities were significantly larger than that induced with sub-maximal exercise, but comparable in patients achieving $>85 \%$ of predicted HR. This underscores the need for an effective test in order to evaluate the right extent of ischemia. ${ }^{19}$

Manganelli et $\mathrm{al}^{20}$ demonstrated that addition of atropine during exercise, in subjects who initially failed to achieve $85 \%$ of maximal predicted HR, was associated with a 20-fold higher probability of achieving target $\mathrm{HR}$ and resulted in the improved ability to detect stressinduced ischemia (47\% in the atropine group vs $29 \%$ in the placebo group). This stepwise approach based on exercise with a pharmacologic addition, when needed, appears to be an "ideal" stress technique, particularly in the "real world" where the extensive prescription of $\beta$ blockers could hamper the diagnostic accuracy of either exercise or pharmacologic stress testing ${ }^{21,22}$ and where withdrawal would be hazardous and beyond the guidelines of recommendation. ${ }^{23}$ In this respect, patients with normal MPI had a very low risk whether or not taking antianginal therapy at the time of stress, but these drugs may lead to an underestimation of the need for 
revascularization by a downgrading of ischemia on MPI. ${ }^{18}$ Therefore, in the setting of prognostic stratification and clinical decision-making, a maximum effectiveness provocative test may be preferable for a more comprehensive assessment of myocardial ischemia and left ventricular dysfunction. $^{4}$

\section{EXERCISE END POINT}

Peak exercise HR has never been intended to be an exercise end point in itself. ${ }^{24}$ Typical angina and/or significant ST-segment depression, drop in blood pressure, or arrhythmias may have already been considered as exercise test end-point for stress MPI, also on the basis that perfusion defect occurs much earlier than angina and ST changes. This is true particularly for diagnostic purposes. Patients with multivessel CAD might have stopped exercise when ischemia was achieved in the worst vascular territory, but not in a territory supplied by a vessel with a less severe stenosis. ${ }^{17}$ In these cases, there may be an underestimation of the degree of ischemia that may influence therapeutic decision. ${ }^{5,6}$ On the other hand, patients who achieved a workload of $\geq 10$ METS but did not achieve $85 \%$ of maximal HR had more ischemia than patients who achieved their target HR, ${ }^{14}$ unmasking chronotropic incompetence, which is predictor of adverse outcome. ${ }^{12}$ Thus, the importance of achieving a target HR should not be overestimated particularly in a symptom- or ST depression-limited exercise ECG. ${ }^{25}$

\section{OPTIMIZATION OF PROGNOSTIC EFFECTIVENESS OF EXERCISE TEST AND MPI}

Despite the well-known limitations of the standard exercise ECG for diagnosing obstructive CAD, this test continues to have enormous prognostic value primarily based on measures of functional capacity, chronotropic response, exercise-induced angina, HR recovery, and ventricular ectopy. ${ }^{12}$ Some authors suggested that additional risk stratification with MPI might be eliminated in individuals who achieve both $85 \%$ predicted HR and adequate exercise level without ischemic ST-segment depression. ${ }^{14}$ On the other hand, while the prognostic power of MPI is well known, SPECT has the major drawback of relative assessment of perfusion. In fact, a considerable proportion of high-risk patients with significant left main CAD may present near-normal MPI, possibly as a result of balanced myocardial ischemia. ${ }^{26}$ In these patients, exercise ECG may provide important diagnostic and prognostic information that may contribute to the appropriate assessment. Although clinical algorithms that integrate ECG and MPI results have not yet been fully established, it appears desirable to use full clinical informative potentials of the two tests. The increases during the exercise of parameters of myocardial oxygen consumption, such as HR and rate-pressure product, may induce progressive myocardial perfusion abnormalities. It is conceivable that quantitative indexes of MPI ischemia disconnected from oxygen consumption evaluation might lead to suboptimal clinical judgments and risk stratification. Therefore, it would be interesting to investigate the value of a standardized integration of the contents of both exercise ECG stress test and MPI. As regards improving the clinical value of exercise ECG, the adjustment of ST depression for HR changes is physiologically logical, ${ }^{27}$ and it appears equally appropriate to combine the degree of ischemia with $\mathrm{HR}$ response (e.g., $>85 \%,>75 \%,<75 \%$ ) to increase diagnostic and prognostic capabilities of MPI. In other words, it would be interesting to know whether the risk of a certain amount of ischemia at a level of $65 \%$ predicted HR is comparable with the same perfusion defect to a level of $>85 \%$ predicted HR, which seems unlikely. ${ }^{25}$

\section{PHARMACOLOGIC STRESS TEST}

In a variable proportion of patients exercise stress test is not feasible and pharmacologic stress represents a valid alternative. At present, up to $50 \%$ of SPECT studies are performed with vasodilators rather than exercise. ${ }^{28}$ Pharmacologic test offers a similar accuracy in unmasking significant $\mathrm{CAD}$, although patients undergoing this stress approach are at a higher risk for subsequent cardiac events even whit normal MPI results. $^{29}$ Unlike exercise ECG, pharmacologic ECG alone seems to have only limited clinical impact. In our view, there is a further potential limitation of vasodilator stress in the individual patient assessment. In evaluating human activities, it is essential to have indicators that monitor the effectiveness of the procedure we are using. For example, we could not assess the efficacy of an antihypertensive therapy without measuring blood pressure, although it had already demonstrated the effect of the drug. Nevertheless, we commonly use a vasodilator as stressor that often lacks of a visible process indicator regarding the presence and degree of pharmacologic effect, such as HR for exercise. It should be also taken into account that everyday substances, such as caffeine, may interfere especially when the vasodilator is used at low doses. In this respect, the use of higher pharmacologic doses may be preferable. Recently, a new selective A2A receptor agonist, regadenoson, has become commercially available for stress MPI. A low risk associated with normal regadenoson SPECT imaging has been demonstrated and, interestingly, patients with normal HR response exhibited a relatively low-annualized total 
mortality. ${ }^{30}$ More studies are clearly needed before we can compare the prognostic value of this new stressor agent with those of exercise stress testing.

\section{CONCLUSIONS}

The new era provides increasing technical, methodological, and clinical options that require choices based on precise goals, even in the logic of care-cost containment. To evaluate the best options, we should often "start from the end," which for MPI means better stratification of risk and guided therapy. For these purposes, the best clinical option seems to be MPI coupled with exercise ECG stress testing, since we are interested to gain global information from the whole procedure. The possibility of adding the two potential makes this choice a "winning couple" which should be well beyond that $50 \%$ of the test. Exercise testing should be maximal in order to better quantify myocardial ischemia. The addition of atropine may contribute to this goal, whereas CZT SPECT cameras seem to improve the accuracy of ischemia detection. Angina and/or significant ST-segment depression may already be considered as exercise test end-point for stress MPI, although the extent of ischemia may be underestimated. Pharmacologic stress may be safely performed in patients who are not candidates for exercise stress with similar MPI accuracy, but with a lower clinical impact. Further interesting perspectives are (1) to correct myocardial ischemia by parameters of oxygen consumption to create more structured and risk-predictive categories; and (2) to investigate the value of standardized clinical algorithms integrating the contents of stress ECG, MPI, and risk factors.

\section{Disclosure}

The authors have indicated that they have no financial conflict of interest.

\section{References}

1. Cuocolo A, Petretta M, Acampa W, De Falco T. Gated SPECT myocardial perfusion imaging: The further improvements of an excellent tool. Q J Nucl Med Mol Imaging 2010;54:129-44.

2. Ladenheim ML, Pollock BH, Rozanski A, Berman DS, Staniloff HM, Forrester JS, et al. Extent and severity of myocardial hypoperfusion as predictors of prognosis in patients with suspected coronary artery disease. J Am Coll Cardiol 1986;7:464-71.

3. Hachamovitch R, Berman DS, Kiat H, Cohen I, Cabico JA, Friedman J, et al. Exercise myocardial perfusion SPECT in patients without known coronary artery disease: Incremental prognostic value and use in risk stratification. Circulation 1996;93:905-14.
4. Sharir T, Germano G, Kavanagh PB, Lai S, Cohen I, Lewin HC, et al. Incremental prognostic value of post-stress left ventricular ejection fraction and volume by gated myocardial perfusion single photon emission computed tomography. Circulation 1999;100: 1035-42.

5. Hachamovitch R, Hayes SW, Friedman JD, Cohen I, Berman DS. Comparison of the short-term survival benefit associated with revascularization compared with medical therapy in patients with no prior coronary artery disease undergoing stress myocardial perfusion single photon emission computed tomography. Circulation 2003;107:2900-7.

6. Shaw LJ, Berman DS, Maron DJ, Mancini GB, Hayes SW, Hartigan PM, et al. Optimal medical therapy with or without percutaneous coronary intervention to reduce ischemic burden: Results from the Clinical Outcomes Utilizing Revascularization and Aggressive Drug Evaluation (COURAGE) trial nuclear substudy. Circulation 2008;117:1283-91.

7. Petretta M, Cuocolo A. Prediction models for risk classification in cardiovascular disease. Eur J Nucl Med Mol Imaging 2012;39: 1959-69.

8. Gimelli A, Bottai M, Giorgetti A, Genovesi D, Kusch A, Ripoli A, et al. Comparison between ultrafast and standard single-photon emission CT in patients with coronary artery disease. A pilot study. Circ Cardiovasc Imaging 2011;4:51-8.

9. Gimelli A, Liga R, Pasanisi EM, Casagranda M, Coceani M, Marzullo P. Influence of cardiac stress-protocol on myocardial perfusion imaging accuracy: The role of exercise level on the evaluation of ischemic burden. J Nucl Cardiol 2015. doi: 10.1007/s12350-015-0101-z.

10. Hendel RC, Berman DS, Di Carli MF, Heidenreich PA, Henkin RE, Pellikka PA, et al. American College of Cardiology Foundation Appropriate Use Criteria Task Force; American Society of Nuclear Cardiology; American College of Radiology; American Heart Association; American Society of Echocardiography; Society of Cardiovascular Computed Tomography; Society for Cardiovascular Magnetic Resonance; Society of Nuclear Medicine. ACCF/ASNC/ACR/AHA/ASE/SCCT/SCMR/SNM 2009 appropriate use criteria for cardiac radionuclide imaging: A report of the American College of Cardiology Foundation Appropriate Use Criteria Task Force, the American Society of Nuclear Cardiology, the American College of Radiology, the American Heart Association, the American Society of Echocardiography, the Society of Cardiovascular Computed Tomography, the Society for Cardiovascular Magnetic Resonance, and the Society of Nuclear Medicine. J Am Coll Cardiol 2009;53:2201-29.

11. Rossen JD, Simonetti I, Marcus ML, Winniford MD. Coronary dilation with standard dose dipyridamole and dipyridamole combined with handgrip. Circulation 1989;79:566-72.

12. Kligfield P, Lauer MS. Exercise electrocardiogram testing: Beyond the ST segment. Circulation 2006;114:2070-82.

13. Myers J, Prakash M, Froelicher V, Do D, Partington S, Atwood JE. Exercise capacity and mortality among men referred for exercise testing. N Engl J Med 2002;346:793-801.

14. Bourque JM, Holland BH, Watson DD, Beller GA. Achieving an exercise workload of $>10$ metabolic equivalents predicts a very low risk of inducible ischemia: Does myocardial perfusion imaging have a role? J Am Coll Cardiol 2009;54:538-45.

15. Duvall WL, Levine EJ, Moonthungal S, Fardanesh M, Croft LB, Henzlova MJ. A hypothetical protocol for the provisional use of perfusion imaging with exercise stress testing. J Nucl Cardiol 2013;20:739-47.

16. Esquivel L, Pollock SG, Beller GA, Gibson RS, Watson DD, Kaul S. Effect of the degree of effort on the sensitivity of the exercise 
thallium-201 stress test in symptomatic coronary artery disease. Am J Cardiol 1989;63:160-5.

17. Iskandrian AS, Heo J, Kong B, Lyons E. Effect of exercise level on the ability of thallium-201 tomographic imaging in detecting coronary artery disease: Analysis of 461 patients. J Am Coll Cardiol 1989;14:1477-86.

18. Brown KA, Rowen M. Impact of antianginal medications, peak heart rate and stress level on the prognostic value of a normal exercise myocardial perfusion imaging study. J Nucl Med 1993;34:1467-71.

19. Mahmarian JJ. State of the art for coronary artery disease: Thallium-201. In: Zaret B, Beller GA, editors. Nuclear cardiology: State of the art and future directions. 2nd ed. St. Louis: Mosby; 1999. p. 237-72.

20. Manganelli F, Spadafora M, Varrella P, Peluso G, Sauro R, Di Lorenzo E, et al. Addition of atropine to submaximal exercise stress testing in patients evaluated for suspected ischaemia with SPECT imaging: A randomized, placebo-controlled trial. Eur J Nucl Med Mol Imaging 2011;38:245-51.

21. Sharir T, Rabinowitz B, Livschitz S, Moalem I, Baron J, Kaplinsky E, et al. Underestimation of extent and severity of coronary artery disease by dipyridamole stress thallium-201 single-photon emission computed tomographic myocardial perfusion imaging in patients taking antianginal drugs. J Am Coll Cardiol 1998;31: 1540-6.

22. Taillefer R, Ahlberg AW, Masood Y, White CM, Lamargese I, Mather JF, et al. Acute beta-blockade reduces the extent and severity of myocardial perfusion defects with dipyridamole Tc99m sestamibi SPECT imaging. J Am Coll Cardiol 2003;42:147583

23. Gibbons RJ, Balady GJ, Bricker JT, Chaitman BR, Fletcher GF, Froelicher VF, et al. American College of Cardiology/American Heart Association Task Force on Practice Guidelines. Committee to Update the 1997 Exercise Testing Guidelines. ACC/AHA 2002 guideline update for exercise testing: summary article. A report of the American College of Cardiology/American Heart Association Task Force on Practice Guidelines (Committee to Update the 1997 Exercise Testing Guidelines). J Am Coll Cardiol 2002;40:1531-40.

24. Wackers FJT. Customized exercise testing. J Am Coll Cardiol 2009;54:546-8.

25. Azarbal B, Hayes SW, Lewin HC, Hachamovitch R, Cohen I, Berman DS. The incremental prognostic value of percentage of heart rate reserve achieved over myocardial perfusion singlephoton emission computed tomography in the prediction of cardiac death and all-cause mortality superiority over $85 \%$ of maximal age-predicted heart rate. J Am Coll Cardiol 2004;44:42330.

26. Berman DS, Kang X, Slomka PJ, Gerlach J, de Yang L, Hayes $\mathrm{SW}$, et al. Underestimation of extent of ischemia by gated SPECT myocardial perfusion imaging in patients with left main coronary artery disease. J Nucl Cardiol 2007;14:521-8.

27. Okin PM, Kligfield P. Heart rate adjustment of ST segment depression and performance of the exercise electrocardiogram: A critical evaluation. J Am Coll Cardiol 1995;25:1726-35.

28. Miyamoto MI, Vernotico SL, Majmundar H, Thomas GS. Pharmacologic stress myocardial perfusion imaging: A practical approach. J Nucl Cardiol 2007;14:250-5.

29. Navare SM, Mather JF, Shaw LJ, Fowler MS, Heller GV. Comparison of risk stratification with pharmacologic and exercise stress myocardial perfusion imaging: A meta-analysis. J Nucl Cardiol 2004;11:551-61.

30. Hage FG, Dean P, Iqbal F, Heo J, Iskandrian AE. A blunted heart rate response to regadenoson is an independent prognostic indicator in patients undergoing myocardial perfusion imaging. J Nucl Cardiol 2011;18:1086-94. 